\title{
Pratiques
}

Linguistique, littérature, didactique

\section{Pourquoi et comment cela change? \\ Standardisation et variation dans le champ des discours scientifiques}

Francis Grossmann

\section{(2) OpenEdition}

\section{Journals}

Édition électronique

URL : http://journals.openedition.org/pratiques/1976

DOI : 10.4000/pratiques. 1976

ISSN : 2425-2042

Éditeur

Centre de recherche sur les médiations (CREM)

\section{Édition imprimée}

Date de publication : 15 juin 2012

Pagination : 141-160

Référence électronique

Francis Grossmann, «Pourquoi et comment cela change ? Standardisation et variation dans le champ des discours scientifiques », Pratiques [En ligne], 153-154 | 2012, mis en ligne le 16 juin 2014, consulté le 30 avril 2019. URL : http://journals.openedition.org/pratiques/1976 ; DOI : 10.4000/pratiques.1976

(c) Tous droits réservés 


\section{Pourquoi et comment cela change? Standardisation et variation dans le champ des discours scientifiques}

\section{Francis Grossmann}

Université de Grenoble III, LIDILEM, E.A. 609

\section{Introduction}

Pourquoi et comment prendre en compte la variation dans le champ des discours scientifiques? Et quel peut être l'intérêt d'une approche variationniste des discours scientifiques? De manière qui peut sembler paradoxale, le postulat de départ développé ici est qu'une prise en compte de la variation est le seul moyen de préserver l'unité d'une macro-catégorie discours scientifique (désormais DS), subsumant les différences disciplinaires et méthodologiques qui clivent les formes d'écritures scientifiques. Le terme de variation englobe deux aspects qu'il vaut mieux différencier : j'opposerai ainsi la diversité liée aux différences de genres, de langues, de cultures, de disciplines, de paradigmes, de méthodologies à la variation interne qui concerne les marges qui peuvent être autorisées - où que certains s'autorisent - par rapport aux normes au sein d'un même genre et au sein d'une même discipline. La thèse développée dans notre contribution peut être résumée de la manière suivante :

- les approches comparatives, notamment dans le courant de la "rhétorique constrastive » (" contrastive rhetoric ») malgré leurs apports, ont jusqu'à présent peu pris en compte la variation interne, ce qui les conduit, de fait, à renforcer les normes existantes en généralisant des tendances observées au sein de certaines cultures ou au sein de certaines disciplines ;

- corollairement, la diversité générique et disciplinaire a été étudiée, parfois de manière assez fine, mais les chercheurs ont eu tendance à considérer disciplines, genres, langues comme des pré-construits; d'où également le caractère faiblement explicatif des différences observées : les différences semblent relever d'une «nature » intrinsèque des disciplines et/ou des 
langues/cultures, sans que soient suffisamment pris en compte l'évolution historique des disciplines et des genres, les poids des institutions, le jeu des influences entre disciplines, et même les parcours individuels. Le point de vue adopté se veut donc essentiellement critique et programmatique, ce qui semble nécessaire au stade actuel.

Dans une première partie, je rappellerai les fondements d'une approche unificatrice des DS. Dans une seconde partie, je résumerai quelques-unes des principales approches qui ont tenté de prendre en compte la variation culturelle, disciplinaire et linguistiques, en soulignant leurs apports, mais aussi en pointant leurs limites.

\section{Une science une... mais divisible}

Un constat : contrairement à ce que l'on pourrait penser, les DS en tant que tels ont, en définitive, été relativement peu analysés dans le cadre de l'analyse $\mathrm{du}$ discours d'inspiration linguistique. On se reportera cependant, dans le champ francophone, aux travaux de Loffler-Laurian (1980, 1983) sur l'expression du locuteur en chimie et en physique, ou, pour les sciences humaines, aux travaux de Pêcheux (réunis par Denise Maldidier en 1990 dans l'Inquiétude du discours), de Maingueneau et Cossuta (1995) sur les «textes constituants», de Guillaumou et Maldidier (1986) sur les textes historiques. L'analyse du discours a privilégié durant de longues années les discours politiques, les discours de presse, ou encore la vulgarisation scientifique parfois assimilée un peu vite aux discours scientifiques. Plus récemment, la recherche didactique s'est intéressée à ce que l'on nomme dans les pays anglophones academic writing et que l'on traduit tant bien que mal en français par écrit universitaire, notion trop large pour être véritablement opératoire ${ }^{(1)}$. Le fait de déplacer le curseur du côté de l'écriture scientifique, et de manière plus générale, des enjeux de connaissance scientifiques présente donc un double avantage : il évite le repli institutionnel qu'implique le terme academic writing et n'envisage les normes universitaires que comme des normes parmi d'autres, la question centrale étant celle des caractéristiques de la discursivité scientifique.

\subsection{Des stéréotypes à une représentation prototypique}

Traditionnellement, les études de la science (Science Studies) ont privilégié les sciences exactes, parce qu'il est entendu que celles-ci incarnent, de la manière la plus typique, les procédés de démonstration et de preuve mis en œuvre dans les démarches que l'on cherche à analyser, dans une optique qui considère que la scientificité se mesure à la capacité à reproduire les mêmes résultats à

(1) L'expression d'écriture de recherche, utilisée en particulier par l'équipe Theodile et par celle du Lidilem (voir par exemple Reuter, 1998 ou De Nuchèze, 1998) présente un avantage certain par rapport à celle d'écrit universitaire, qui semble confiner la recherche au sein de l'institution académique, mais elle ne l'intègre pas de manière suffisamment visible au champ des Science Studies, actuellement dominé par la sociologie des sciences. 
partir des mêmes prémisses ou à partir des mêmes données expérimentales. Cependant, on a pu constater (Rosental, 2009) que le terme démonstration renvoie à des pratiques et des objets eux-mêmes très différents, y compris au sein des sciences déductives. La priorité accordée aux sciences exactes a eu comme corollaire l'idée que leurs procédures se situaient en dehors du champ social. Les normes qui se sont progressivement imposées aux textes scientifiques semblent reposer sur un postulat d'unicité, fondamentalement lié à l'universalité du raisonnement scientifique, ou plus largement encore, à l'existence des principes généraux régissant la cognition humaine, dont témoigne par exemple l'universalité des règles logiques, elles-mêmes au-dessus des contingences de l'expression linguistique. C'est aussi ce que rappelle Sabine Schwarze $(2008: 4)$ :

« Sur cette ligne universalisante on continue à définir le style scientifique dans le cadre d'une conception européenne idéalisée, qui voit dans la science essentiellement le reflet de la vérité, et dans la langue utilisée par un auteur faillible, un obstacle à l'expression de ces vérités scientifiques. »

Cette conception conduit à postuler un modèle unique de l'activité scientifique, identifié aux représentations caractéristiques des sciences expérimentales. Cependant, l'unicité proclamée de la science ne masque pas longtemps la diversité des pratiques scientifiques, et moins encore les partitions entre les différents domaines scientifiques, qui fondent aussi des partages disciplinaires institutionnalisés, induisant des dichotomies telles que sciences exactes $v s$ sciences humaines, sciences fondamentales $v s$ sciences appliquées, etc. à quoi correspondent aussi des différences évidentes au plan des objets, des méthodes, des enjeux cognitifs visés, et tout autant, des pratiques d'écriture. Quoi de commun en effet entre un article d'ethnologie, qui peut adopter la forme d'un récit, et un article de biologie médicale qui se plie au format $\mathrm{IMRaD}^{(2)}$ ? Il semble plus efficace de comparer des disciplines appartenant à une même famille d'écrits scientifiques, plutôt que de tenter de rapprocher artificiellement des écrits que tout semble opposer. Cet argument mérite cependant d'être nuancé, dans la mesure où la variation traverse l'intérieur des disciplines et des communautés scientifiques, soit à travers la fragmentation en sous-disciplines (psychologie clinique $v s$ psychologie cognitive), soit à travers l'usage des méthodologies mêmes : la phonétique d'aujourd'hui se veut expérimentale, mais relève bien des sciences humaines. Il reste commode d'établir deux familles principales de modèles de scientificité ${ }^{(3)}$ :

- les modèles de prédiction (ex. physique) fondés sur une démarche hypothético-déductive, et dans les sciences empiriques, sur la démarche expé-

(2) Acronyme pour Introduction, Methods, Results and Discussion. Comme le remarque Pontille (2007), ce format imposé dans les disciplines expérimentales n'est pas une simple feuille de style, mais également un dispositif scénique permettant l'expression de la preuve.

(3) Qui se traduit également, comme le fait remarquer J.-L. Fabiani, que je remercie de sa relecture attentive, à travers l'opposition classique entre sciences nomothétiques et sciences herméneutiques. 
rimentale ; épistémologiquement, ils se plient à l'exigence de la falsification poppérienne ;

- les modèles herméneutiques (ex. histoire) pour lesquels la validation passe par la vérification des sources, et la vérification de leur fiabilité; la connaissance du contexte guide l'interprétation et le chercheur s'appuie sur des traditions interprétatives pour comprendre le sens d'un détail dans l'ensemble, etc.

A chacun de ces deux types correspondent des formes de validation spécifiques, les caractéristiques de l'écrit produit dépendant en partie du modèle de scientificité implicite ou explicite adopté par le chercheur. Il ne s'agit pas de nier le fait qu'un saut qualitatif oppose sciences de la nature et sciences de l'homme, la part «interprétative » prenant dans le second cas une importance beaucoup plus grande. La nuance que l'on peut apporter, c'est que souvent, dans la science moderne, des modèles et des méthodologies croisés ou complémentaires sont mobilisées pour mieux appréhender la complexité des objets à traiter. Un archéologue ou un psychologue fait appel à des dispositifs expérimentaux pour vérifier certaines de ses hypothèses. La médecine se fonde en grande partie sur des données expérimentales, mais comporte aussi une sémiologie des symptômes et sur un « savoir-faire » qui la rapproche dans certains cas de l'herméneutique et même d'un «art», etc. Et même une science expérimentale telle que la physique comporte également une part interprétative qui exige la validation de sources liées à l'observation, voire même une certaine part de sémiologie, étant donnée la part croissante de l'image dans l'instrumentation.

Quel que soit le modèle de scientificité sur lequel on s'appuie, le fait d'emporter la conviction des pairs reste une opération compliquée, qui ne repose pas sur la seule clarté de la démarche scientifique mobilisée et sur le statut de la preuve fournie. Les procédures d'adhésion et de persuasion à une nouvelle théorie scientifique sont liées à la construction de nouvelles communautés scientifiques ( $c f$. Kuhn, 1962 ; Feyerabend, 1975), lesquelles donnent naissance à des ruptures, des débats, des polémiques... On peut insister, comme le fait Bourdieu (1984), sur la concurrence dans un champ scientifique en se fondant sur une vision agoniste, non irénique de la science (voir aussi Olivesi, 2007). Il est clair que les disciplines sont structurées par ces oppositions, qui manifestent leurs évolutions internes, mettent en évidence les changements de paradigme et de méthodologie, et pèsent aussi directement sur leur structuration discursive. Touati (2010) montre par exemple que les controverses qui se sont développées en phonétique au moment où s'est renouvelée la discipline à partir de procédures expérimentales et de nouveaux instruments ont influencé la structure textuelle même de la thèse, provoquant des débats au sein des jurys, certains phonéticiens traditionnels restant attachés à la " thèse somme », éloignée de la nouvelle conception des expérimentalistes. Si l'on adopte cette vision à la fois holistique et variationniste de l'activité scientifique, le prototype qui peut asseoir une représentation commune et unificatrice de l'activité scientifique, peut s'énumérer en quatre points : 
- Existence d'un raisonnement (raisonnement hypothético-déductif et/ou inductif) ;

- Existence d'un dispositif méthodique permettant le recueil et le traitement d'informations et/ou de données, quelle que soit par ailleurs la nature de ce dispositif;

- Existence d'un système de preuve : ces preuves, qui peuvent elles-aussi être de nature très différentes, requièrent un système argumentatif visant l'adhésion du public scientifique ;

- Existence de résultats et communication de ces résultats au sein d'une communauté de pairs sous des formes standardisées.

Ces quatre constituants laissent de côté des aspects très importants caractérisant l'activité scientifique, en particulier tout ce qui concerne le questionnement, l'intuition, l'imagination, la création qui appartiennent également l'activité artistique et littéraire : bien que fondamentaux, ils ne figurent donc pas en tant que tels dans la représentation prototypique de l'activité scientifique.

\subsection{L'idée d'un style scientifique « universel »}

Schwarze (2008) rappelle que le sociologue Gusfield dans son ouvrage sur la rhétorique de la science, proposait comme définition du style scientifique l'absence de tout style, donc le «non-style ». Certains auteurs, signale encore Schwarze, ont tenté de définir plus positivement un style scientifique universel. C'est le cas par exemple de Kocourek qui le résume à travers quatre traits principaux : systématisation notionnelle; précision sémantique avec un tabou des métaphores ; neutralité émotive et affective avec un tabou du « moi »; économie formelle accompagnée d'un tabou portant sur le narratif. Le premier critère se fonde sur le découpage du champ notionnel considéré et concerne également les techniques, et de manière générale, tout domaine spécialisé ; le critère de précision sémantique est sans doute un critère définitoire relativement efficace, mais il est insuffisamment discriminant (il est peut-être encore plus requis dans le domaine technique que dans le domaine scientifique); le tabou des métaphores renvoie à une conception ornementale des métaphores, alors que les métaphores ont souvent une fonction interprétative ou heuristique et sont parfois utilisées dans le style scientifique. De même, le critère de neutralité émotive et affective se fonde sur la nécessité de l'effacement énonciatif, or cet effacement n'entraîne pas celui du sujet épistémique ; l'idée que le sujet épistémique ne se confond pas avec la personne individuelle du chercheur n'oblitère donc pas la nécessité du positionnement scientifique. Enfin, le tabou sur le narratif s'applique mal aux sciences humaines, et même dans les sciences exactes, certaines formes de récit peuvent être trouvées.

Il est donc difficile, à partir de tels critères, de définir un style scientifique universel, sans doute proprement introuvable. Cela n'empêche pas de reconnaître la tendance au rapprochement des formes discursives scientifiques, ni à nier les influences réciproques liées à la mondialisation de la science, au développement de normes, qui se traduisent par certaines tendances communes, comme l'effacement énonciatif, ainsi que la mobilisation d'un lexique « trans- 
disciplinaire » propre à toute communication scientifique. L'utilisation d'un tel lexique commun, par exemple l'utilisation de mots-clés tels que postulat, hypothèse (Cavalla \& Grossmann, 2005) par différentes disciplines ne garantit évidemment pas que l'on parle des mêmes choses. Il y a un cependant un air de famille entre ces différents emplois, qui colore l'ensemble des discours scientifiques. Certains auteurs, comme par exemple Spillner (1989) parlent de conventions de style (Stilconventionen) qui seraient différentes selon les languescultures. Une bonne partie des travaux issus du courant de la rhétorique contrastive - nous y revenons plus loin - a eu pour but d'infirmer le point de vue universaliste, en insistant sur la relativité des styles scientifiques, par rapport aux normes des langues-cultures, et des disciplines.

\subsection{La standardisation de l'écriture scientifique et ses limites}

On peut observer un double mouvement contradictoire : d'une part, dans certaines disciplines, notamment en Sciences Humaines et Sociales, ainsi que dans certaines traditions qui ne recouraient pas aux marques personnelles (comme en France), il y a une «personnalisation » apparente de l'écrit scientifique, avec l'utilisation plus grande de formes personnelles (pronoms de la $1^{\text {re }}$ personne, y compris le « je ») et donc un « effet de présence » de l'auteur. Mais inversement, le développement du plan IMRaD, dans les disciplines scientifiques d'abord, mais aussi dans certaines sciences humaines et sociales, conduit aussi à accentuer la dépersonnalisation (déjà souvent présente à travers l'effacement énonciatif classiquement évoqué pour l'écrit scientifique).

\subsubsection{La structure IMRaD}

Les effets du plan IMRaD ont été bien analysés par Pontille (2007 : 13), qui montre en quoi ce format se présente comme une forme de structuration du travail collectif tout en pointant ses limites comme instrument d'évaluation de la pratique scientifique :

«Le format IMRAD participe pleinement de la structuration d'une écologie professionnelle singulière au sein des activités scientifiques : la communauté des chercheurs regroupés autour de procédures expérimentales. La forte légitimité de ces dernières constitue une ligne de tensions, particulièrement prononcée dans certaines sciences sociales, entre les partisans de la démarche expérimentale dont les résultats sont appuyés sur la validité des chiffres et ceux de la tradition herméneutique qui visent la construction d'intelligibilité passant par le sens des mots ».

L'aboutissement de cette structuration oppose le statut de l'écrit dans les sciences sociales et les sciences expérimentales : en effet l'écrit se donne à lire, dans le premier cas, comme un texte « à part entière », travaillant la forme textuelle dans sa dimension mathétique, alors que, dans le second, il n'est plus qu'un simple compte-rendu des connaissances produites, la partie « discussion » concentrant, au moins en apparence, le débat argumentatif, mais seule- 
ment à l'issue des résultats obtenus. Cette disjonction pèse elle-même dans le statut de l'auteur, qui peut conserver ou non une présence individuelle « absolue » au sens littéraire du terme ou bien n'avoir qu'une présence minimale, l'écrit n'étant dans ce cas que l'étalon standardisé de conformité à un groupe professionnel, et construisant bien souvent un auteur collectif(Pontille, 2004). Comme le rappelle encore Pontille, si l'argumentation personnelle semble s'amoindrir, le poids argumentatif ne disparaît pas. Il est, dans le dispositif IMRaD, endossé par la structure textuelle elle-même qui acquiert de ce fait une fonction symbolique, rendant possible des effets d'imposition, voire de contrefaçon (la structure textuelle donnant l'impression à elle seule de " faire science »). Les études sur les cultures disciplinaires (voir en particulier Fløttum, 2007) montrent que ce type de format reste cependant pour l'instant peu fréquent en linguistique et même en économie. Il faudrait sans doute mesurer plus précisément le degré de pénétration actuel dans les différentes disciplines du format IMRaD ou d'autres formats standardisés.

\subsubsection{L'effacement énonciatif}

La règle d'effacement énonciatif semble consubstantielle à l'écriture scientifique. Cependant, comme nous le verrons à propos de la rhétorique contrastive, l'interprétation des marques signalant le retrait de l'énonciateur est relativement complexe, et les systèmes varient suivant les langues et les disciplines. Il est délicat de s'intéresser au passif, au we au $I$, au Je ou au Nous sans analyser en finesse le rôle des indices personnels, en fonction de l'histoire d'une langue. L'utilisation du on français en fournit un exemple éclatant : sa plasticité en fait un instrument permettant tantôt l'effacement du scripteur et tantôt la connivence, avec implication du lecteur (Fløttum, Jonasson \& Noréen, 2007). Mais l'on peut constater, quelle que soient les disciplines et les champs de recherche, une tendance générale à cet effacement. Ces constats conduisent à refuser d'autonomiser des domaines qui seraient présentés a priori comme radicalement différents, tant du point de vue des démarches que de l'activité d'écriture.

\section{Les approches contrastives de l'écrit scientifique : apports et limites}

\subsection{La rhétorique contrastive}

La comparaison des spécificités de l'écriture scientifique selon les langues et les cultures a donné lieu à de nombreux travaux intéressants et documentés ces dernières années. Ces différents travaux s'inscrivent surtout dans les pays anglo-saxons, mais aussi en Allemagne et plus récemment dans d'autres pays, dans un important courant se revendiquant, depuis les recherches pionnières de Robert Kaplan $(1966,1972)$, de la « rhétorique contrastive ». Cette approche est née du constat des limites de la linguistique générale et constrastive, qui 
semblait se cantonner au niveau phrastique sans prendre en compte les stratégies textuelles ${ }^{(4)}$. Les auteurs associés à la rhétorique contrastive défendent l'idée, difficilement contestable en elle-même, que, dans la mesure où l'écrit - et pour ce qui nous occupe ici l'écrit scientifique - est fondamentalement un phénomène culturel, son organisation (entendue essentiellement comme superstructure, organisation textuelle, $c f$. l'organisation paragraphique, étudiée par Kaplan) est conditionnée par les caractéristiques culturelles spécifiques, relevant du contexte propre à la société qui les a produites. Dans sa thèse, Poudat (2008: 62-63) synthétise cet apport de la rhétorique contrastive, en se focalisant plus spécifiquement sur le courant ESP («English for scientific purpose »). Elle rappelle que, selon ces recherches, les articles anglo-saxons seraient généralement « orientés vers le lecteur »; l'emploi du style dialogique (ou reader-friendly) présenterait l'article scientifique comme un contrat à négocier, dans lequel on donnerait au lecteur le sentiment de participer; ce ne serait pas le cas, par exemple, de la tradition intellectuelle germanique, qui se fonderait sur un style monologique qui valoriserait davantage la créativité de l'auteur. On ne peut s'empêcher de penser que la rhétorique contrastive continue, pour l'essentiel, à s'appuyer, implicitement ou explicitement, sur la notion de style intellectuel, lié principalement à des traditions nationales, considérées comme autonomes et concurrentes. Johann Galtung (1985), partant d'une observation sociologique des relations avait identifié quatre styles intellectuels ( «teuton, saxon, gaulois, nippon »). Cette typologie est évidemment très critiquable, comme le montre bien S. Schwarze (2008) à propos du style " gaulois », en mettant en évidence la fragilité de l'argumentation de Galtung, appuyée sur le rôle de l'élégance, des figures rhétoriques et des jeux des jeux de mots qui seraient les moyens décisifs de persuasion et sembleraient ainsi exclure les vertus universelles du discours scientifique.

Contrairement aux travaux de Galtung, les études de Clyne $(1987,1991)$ reposent sur une base empirique (bien que limitée). Le corpus de 1991 comprend 52 textes, dont la moitié écrite par des Allemands et l'autre moitié par des scientifiques anglophones. Clyne compare les différences organisationnelles des textes académiques anglo-saxons et allemands, et veut montrer que si les premiers ont tendance à privilégier les développements linéaires, l'explicitation préalable des termes employés ou le style reader-friendly, les seconds préfèrent les développements digressifs et la non explicitation des termes, liée à l'idéalisation du savoir. Chez les Anglo-Saxons, ce serait l'auteur qui devrait s'efforcer de rendre son texte lisible, tandis que ce sont les lecteurs qui devraient faire l'effort d'adaptation dans les communautés germanique et française. Diverses études (par ex. Saville-Troike, 1982) vont encore plus loin dans la généralisation, et décrivent par ailleurs la tendance qu'auraient les cultures de l'ouest, considérées comme " plus individualistes », à employer des styles plus directs et explicites, tandis que les sociétés orientales insisteraient davantage sur les valeurs collectives et l'harmonie du groupe, employant un style in-

(4) Ces stratégies textuelles sont vues comme enracinées dans la culture, ce qui donne un sens très spécifique au terme rhétorique, par rapport à son emploi plus classique (pour une présentation de ce courant, voir Connor, 2002). 
teractif plus défensif et plus imprécis. Les reproches d'ethnocentrisme, parfois émis à l'encontre des approches de la rhétorique contrastive, se trouvent ici pleinement justifiés, étant donné le caractère très global et souvent discutable de telles affirmations. Surtout, la variation est renvoyée à des spécificités culturelles globales, au lieu d'être analysée en fonction de facteurs historiques, sociologiques, ou à partir d'une étude fine des contextes de production, ainsi qu'à partir d'une analyse circonstanciée des traditions orientant la réception. D'autres auteurs ont eu cependant une approche plus subtile et mieux étayée empiriquement. Schwarze (2008 : 10), synthétisant les apports de la germanistique interculturelle signale que selon Schröder (2005) et Gnutzmann (1989) les différences de détermination des textes par des traditions discursives particulières s'expliqueraient soit par la discipline, soit par les paradigmes scientifiques dans lesquels s'inscrivent les scientifiques. Gnutzmann (1989) avait cantonné son approche au facteur disciplinaire en soulignant les différences entre les disciplines scientifiques de caractère plutôt transculturel et celles qui seraient marquées par les modèles discursifs spécifiques d'une culture linguistique particulière. Reutner $(2010$ : 82) insiste de son côté sur le fait que les traditions nationales sont moins prégnantes que les traditions culturelles, mais aussi les disciplines, sous-disciplines et écoles (voir aussi sur ce point Fløttum, 2004 : 403 ; Fløttum et al., 2007).

\subsection{Les recherches sur le métadiscours}

Dans le prolongement immédiat des recherches menées en rhétorique contrastive, certains auteurs, notamment l'anglais Ken Hyland, se sont plus précisément intéressés - uniquement pour l'anglais en ce qui concerne Hyland - à l'usage différencié, selon les disciplines, et parfois, de certains types de marques. Hyland utilise le terme de métadiscours comme une étiquette subsumant l'ensemble des marques impliquant une forme de réflexivité du scripteur dans le cadre de la négociation de l'interaction avec les lecteurs appartenant à une communauté spécifique. Les marques de métadiscours sont donc fort nombreuses et très diverses. Plusieurs classifications sont reprises et analysées dans Hyland (2005). La plupart des typologies partent de la classification établie par Vande Kopple (1985), à partir de la distinction classique faite par Halliday (1973) entre deux macrofonctions du langage, l'une appelée « textuelle », la seconde « interpersonnelle ». La première fonction (à quoi correspondrait le métadiscours textuel) permet le développement de stratégies rhétoriques du scripteur permettant la mise en texte de l'expérience de manière cohérente; c'est à partir de lui que se construit la structure textuelle. La seconde, correspondant, au métadiscours interpersonnel, concerne les aspects interactionnels et évaluatifs de la présence de l'auteur dans son discours. Cette deuxième catégorie de marques construit plus directement la figure de l'auteur. Les recherches récentes ont cependant tendance à relativiser cette opposition, à partir du constat que l'aspect interactionnel est central dans la définition du métadiscours, et que les buts rhétoriques ne peuvent être distingués clairement de cet aspect interpersonnel. Si l'on ne peut que souscrire à cet infléchissement, 
on voit aussi que tout ou presque peut devenir métadiscours, et l'on rencontre la même difficulté qu'avec la notion de métalangage ${ }^{(5)}$.

Dans une approche fonctionnelle, la notion de métadiscours garde cependant un intérêt : pour Hyland, le métadiscours a une fonction importante de guidage, et permet de rendre un texte reader friendly ${ }^{(6)}$, mais il joue également un rôle social en facilitant l'insertion du texte dans un contexte donné, et en permettant la bonne réception du message, dans le cadre de la communauté de discours concernée. Dans le discours scientifique, le métadiscours contribue ainsi à placer de manière pertinente la voix du scripteur, entre assurance et retenue, et à établir un lien de confiance avec son public. Se situant dans une perspective clairement pragmatique et fonctionnelle Ken Hyland distingue deux dimensions. La dimension interactive concerne la manière dont le scripteur anticipe les attentes et les connaissances préalables du lecteur, et la manière dont l'organisation du texte répond aux besoins spécifiques d'un lectorat particulier ${ }^{(7)}$. La dimension interactionnelle, quant à elle, prend en compte les moyens à travers lesquels le scripteur conduit l'interaction en glosant ou en commentant certains aspects de son propre message ; les buts du scripteur sont ici de rendre ses intentions explicites et d'impliquer les lecteurs ; cette dimension est donc principalement dialogique, évaluative et argumentative ; les marques impliquées dans cette dimension sont les modalisateurs ; les marqueurs de positionnement qui permettent d'exprimer le point de vue du scripteur (ex. malheureusement, heureusement, remarquable...) ; les marques de première personne, pronoms ou adjectifs possessifs (Je, moi, mon, ma, nous académique " exclusif», notre ...) ; les marqueurs d'implication qui visent à « enrôler» le lecteur et à l'impliquer dans un raisonnement, une démonstration (ex. nous inclusif, marques de seconde personne, you may notice, etc.).

En ce qui concerne les différences d'emploi des marqueurs de métadiscours selon les langues-cultures, on dispose de quelques études (résumées dans Hyland, 2005 : 117-118). Bloch et Chi (1995) par exemple, examinent dans 120 articles écrits en anglais et en chinois les marques évidentielles et la manière dont les auteurs citent leurs sources. Contrairement à ce qui est dit souvent (les Chinois suivraient de manière non critique la tradition confucéenne, privilégiant des valeurs de transmission aux valeurs critiques de la tradition occidentale, qui valoriserait quant à elle la nouveauté et la créativité), Bloch et Chi montrent que les choses se présentent de manière plus complexe : par exemple, si dans l'ensemble, les Anglo-Saxons utilisent davantage de marques évidentielles, les fonctions rhétoriques sont les mêmes dans les deux cas,

(5) Rappelons le mot de Lacan, selon lequel il n'y a pas de métalangage, c'est-à-dire de point de vue extérieur au langage ; le métalangage est partout puisqu'en définitive la mise en œuvre langagière inclut toujours un point de vue sur son propre fonctionnement.

(6) Notons que cette notion est idéologiquement chargée et très relative : en fonction du contexte culturel ce qui est présenté comme « readerfriendly » aux uns apparaîtra comme didactisme pesant et atteinte aux «droits du lecteur» aux autres.

(7) Les marqueurs utilisés permettent de signaler les transitions textuelles, de mettre en évidence la structure textuelle, de renvoyer le lecteur à d'autres endroits du texte; de préciser la source d'une idée, d'un point de vue ; d'expliciter ou de reformuler un élément textuel à l'aide de marqueurs de reformulation. 
ce qui ne confirme pas le postulat évoqué précédemment. Par ailleurs, le nombre de références dont le chercheur critique le contenu (critical citation) est relativement faible pour l'ensemble, même s'il est plus élevé, en anglais, pour les sciences sociales, et en chinois, pour les sciences physiques. Comme le souligne Hyland (2005 : 118), ces résultats montrent la difficulté de faire des généralisations sur des bases culturelles dans le domaine de la rhétorique textuelle. Les besoins sociaux font évoluer les pratiques, qui ne correspondent pas aux stéréotypes. Par ailleurs, comme souvent, il faut adopter le principe du rasoir d'Occam, qui privilégie les explications simples aux explications complexes : s'il y a moins de références nouvelles dans les textes chinois, c'est avant tout parce que l'accès aux sources de connaissances scientifiques, est, pour l'instant encore, plus difficile. Cela ne signifie pas, bien évidemment, qu'il n'existe pas des différences culturelles, mais il est nécessaire alors de les considérer dans une perspective dynamique, en fonction des évolutions historiques et sociales. Les différences observées d'une étude à l'autre (par ex. les résultats de ValeroGarcés, 1996 pour l'anglais et l'espagnol ne sont pas confirmés dans Moreno, 1997, qui, étudiant les marques métatextuelles mettant en évidence la structure causale trouve, d'un point de vue quantitatif, davantage de similitudes que de différences entre les deux langues cultures).

\subsubsection{Les approches orientées vers l'énonciation et les marques de la subjectivité}

L'intérêt du projet KIAP ${ }^{(8)}$ piloté par Kjersti Fløttum (cf. Fløttum, 2007) outre le fait qu'il s'agit d'une des premières recherches en la matière effectuée sur un corpus relativement important d'articles scientifiques dans différentes langues (anglais, français, mais aussi norvégien), est de deux ordres. D'une part, dans un contexte de recherche souvent dominé par des aspects concernant la superstructure textuelle, ces chercheurs sont partis d'un point de vue énonciatif ; ils ont utilisé une large palette de marques linguistiques. D'autre part, ils ont cherché à hiérarchiser l'importance des différents types de facteurs culturels ; partant de trois disciplines différentes (médecine, économie politique et linguistique) dans un corpus comprenant environ trois millions de mots, leurs résultats semblent montrer que la discipline primerait sur l'appartenance linguistique en matière d'identité culturelle. Pour aboutir à ces résultats, une série de catégories d'indices ont été utilisés : pronoms, pronoms indéfinis, marques de métadiscours, références bibliographiques, constructions polyphoniques, marqueurs de modalités épistémiques.

\section{Le poids des disciplines et des cultures nationales}

Les résultats obtenus montrent que les chercheurs médecins écrivent peu à la première personne et recourent massivement aux formes passives ; les chercheurs économistes sont relativement présents dans leur texte, notamment à

(8) L'acronyme traduit renvoie à « Identité culturelle dans le discours scientifique : nationale $v s$ disciplinaire $»$. 
travers des marques de métadiscours (Dans la section 3, nous considérons...), cependant leur présence reste modeste, les linguistes assumant davantage un point de vue affirmé - voire polémique -, entrant dans le cadre d'une argumentation. En ce qui concerne les différences liées à la langue-culture, ces chercheurs confirment certains des constats déjà formulés par d'autres auteurs appartenant au courant de la rhétorique contrastive : les auteurs des articles publiés en anglais seraient plus présents et assureraient le guidage du lecteur, annonçant de manière précise et explicite ce qui va être traité. Ils seraient également relativement polémiques, et dans le cas des articles rédigés par un seul auteur, ils utiliseraient souvent les pronoms de première personne du singulier. Les auteurs norvégiens, également voire plus polémiques, utiliseraient cependant une voix plus collective. En contraste, les auteurs des articles français seraient moins explicitement présents, le guidage des lecteurs restant relativement rare, et la polémique plus implicite qu'explicite. La tendance chez les auteurs français serait d'utiliser le pronom on plutôt que le je ou le nous, comme dans « on peut constater». On retrouve aussi (Dahl, 2004) l'idée que l'anglais et le norvégien seraient représentatifs de cultures qui mettent au premier plan la responsabilité du scripteur, tandis que le français serait emblématique d'une culture plaçant au centre la responsabilité du lecteur. Cette conclusion nous semble à nuancer parce qu'elle a tendance à mettre sur le même plan les routines propres à une langue-culture et les stratégies explicites ou implicites utilisées par les auteurs. Plus convaincant est le constat selon lequel, du fait que certaines disciplines telles que l'économie et la linguistique présentent une structure textuelle moins formalisée que les disciplines régies par le format IMRaD (comme la médecine), l'influence des langues-cultures peut s'y exercer de manière plus importante.

Certains des résultats les plus intéressants (Fløttum, 2007) concernent la répartition, selon les langues-cultures et les disciplines : les marques métatextuelles seraient utilisées davantage en économie qu'en linguistique, davantage en linguistique qu'en médecine. Au plan linguistique, elles seraient utilisées davantage en anglais qu'en norvégien, et davantage en norvégien qu'en français. Etant donné ce qui est englobé dans la catégorie des marqueurs métatextuels, il est en définitive assez difficile de savoir ce qui peut expliquer la variation, l'interprétation restant toujours délicate. Un élément intéressant avancé (p. 168) en ce qui concerne la variation disciplinaire repose sur les normes rédactionnelles : les médecins utilisant massivement le plan IMRaD (contrairement aux linguistes et économistes) n'ont pas besoin de marques métatextuelles pour aider le lecteur à se repérer dans l'article; on note au passage le fait que la fréquence de certaines marques métatextuelles est liée au mode de structuration du texte et à son organisation ; cela relativise l'approche culturelle traditionnelle privilégiant la responsabilité du lecteur ou la responsabilité de l'auteur, puisque c'est en définitive la standardisation de certaines pratiques d'écriture qui est ici en cause.

Parmi les autres marques ayant donné lieu à enquêtes quantitatives, on peut noter :

- l'usage de la première personne : le français utilise le moins, par rapport 
à l'anglais et au norvégien la première personne comme sujet pour renvoyer à l'auteur du texte ; mais il faut noter que le $\mathrm{ON}$ français a des usages beaucoup plus variés que les indéfinis correspondant en anglais et en norvégien ; quand il utilise la première personne, le français préfère le nous académique ;

- les connecteurs adversatifs : le norvégien utilise davantage d'adversatifs que l'anglais et le français ; mais il faut noter que l'enquête a pris seulement en compte but et mais(et leur équivalent norvégien) ;

- les références aux travaux d'autrui : un résultat intéressant concerne le nombre de références bibliographiques : l'anglais et le norvégien comportent beaucoup plus de références bibliographiques ; l'hypothèse explicative, pour l'anglais, est que la communauté discursive est beaucoup plus large, et donc le nombre de travaux potentiellement plus important; pour le norvégien, il est tourné depuis longtemps vers l'extérieur.

\section{Les différences entre experts et néophytes}

Toujours dans une perspective énonciative, certains travaux insistent davantage sur les différences entre rédacteurs expérimentés et néophytes. Ainsi, pour Boch et Grossmann (2002) le discours d'autrui sert essentiellement chez les néophytes à introduire le propos à donner une définition et surtout à illustrer/appuyer une affirmation. Il n'est guère utilisé pour marquer son appartenance à une école, ou à un courant de pensée ou encore pour se démarquer d'une position exprimée par un auteur, enfin pour rappeler l'état d'une question ou d'une problématique Rinck, Boch, Grossmann (2007) montrent également les différences qui opposent les doctorants aux autres chercheurs dans les références au discours d'autrui : on trouve chez eux moins de noms d'auteurs, moins de références à des courants particuliers, pratique réservée aux pratiques expertes.

\section{La diversité des discours selon les genres}

En ce qui concerne les différences internes aux genres scientifiques, certaines études, liant lexique et énonciation, ont fait des constats intéressants, par exemple en ce qui concerne le marquage de l'opinion : Tutin et al. (2009) signalent que, de manière attendue, on trouve davantage de marques d'opinion dans les mémoires d'HDR que dans les autres sous-genres ; de manière plus surprenante, le marquage de l'opinion apparaît plus net dans les communications écrites autres que les articles stricto sensu. Tutin (2010) montre que de manière tendancielle, plus les verbes expriment un positionnement marqué, par exemple, les verbes d'opinion, moins ils sont pris en charge par le locuteur : elle remarque ainsi que les pronoms renvoyant à l'auteur seul (qu'il s'agisse d'un je ou d'un nous ou d'un on de modestie), renvoient surtout aux verbes indiquant un apport scientifique ou une intention, alors que les verbes de positionnement fort (verbes d'opinion) sont plus souvent introduits à l'aide d'un pronom incluant la communauté de discours (nous et on exclusifs) et sont souvent forte- 
ment modalisés (on peut penser que...). Cette étude montre bien la nécessité de ne pas se focaliser sur une seule marque mais de prendre en compte des systèmes ou des micro-systèmes. Le corpus Scientext, à partir duquel sont fournis ces résultats permet d'obtenir facilement des statistiques selon les disciplines et les sous-genres, même si la question de l'interprétation reste toujours délicate et suppose une analyse des contextes de production.

\section{Vers un modèle multidimensionnel pour analyser la variation des DS}

Alors, pour finir, comment penser la variation ? Et quelles catégories peuton mobiliser pour la décrire ? Nous avons vu les apories auxquelles conduit l'idée d'un modèle universel de l'écriture scientifique. La standardisation, qui s'est développée à travers des normes précises dans les disciplines expérimentales, si elle a influencé certains secteurs des sciences humaines et sociales, reste cependant encore limitée. Elle ne pourra guère progresser sans faire courir le risque d'un aplatissement des épistémologies disciplinaires. Cependant, l'intégration de procédures de validation issues des sciences expérimentales, lorsqu'elle est justifiée dans le cadre d'autres disciplines, conduit inévitablement à mettre en avant des éléments de présentation de la preuve qui correspondent à des protocoles standardisés. La hiérarchisation des éléments de preuve et la compréhension de leur statut différent au sein d'un même texte complexifie sans doute les modèles textuels en sciences humaines et sociales et conduira sans doute à faire évoluer ceux-ci. Mais nous avons aussi entrevu les difficultés auxquelles sont confrontées certaines approches de la variation, avec ce nouveau paradoxe : alors que l'étude de variation, telle qu'elle est effectuée par les sociolinguistes, aboutit généralement à une remise en cause critique des catégories, linguistiques mais aussi plus globalement, des impensés philosophiques, historiques, ou sociaux généralement évacués par la grammaire, la prise en compte de la variation pour les discours scientifiques a eu parfois un effet régressif, en confortant les préjugés ethnocentristes, voire des stéréotypes culturels proches d'une folk sociology. Les discours scientifiques sont ainsi très rarement catégorisés à partir de critères prenant en compte les variables historiques, économiques ou sociales (la variable " genre », donc la diversité plutôt que la variation, a été davantage travaillée). L'étude de la variation telle qu'elle a été entreprise notamment sous la bannière de l'English for Specific Purposes entérine aussi globalement le partage entre la langue dominante (l'anglais considérée comme une lingua franca) et les langues dominées (toutes les autres). Cela ne serait en définitive qu'une constatation réaliste de la situation existante, mais les spécialistes de rhétorique contrastive, pour une bonne part, spéculent sur les caractéristiques de langues-cultures étrangères décrites à partir de postulats issus de leurs propres filtres culturels. Avant de présenter, en guise de conclusion, quelques unes des dimensions qui me semblent permettre de penser la variation dans les genres scientifiques, je vais tenter de résumer les principes permettant de remettre « sur ses pieds » l'étude variationniste. 


\subsection{Principes de base d'une approche variationniste}

Une telle approche, dans l'état actuel, se doit avant tout de privilégier une approche descriptive en évitant les termes globalisants (ex. "styles intellectuels »), chargés idéologiquement ou comportant des jugements de valeur ( «reader friendly », etc.). Un second principe important, et aujourd'hui mieux compris, réside dans le fondement empirique qu'apporte l'appui des corpus ; les corpus comparables sont extrêmement difficiles à constituer et il reste encore beaucoup de travail à accomplir pour permettre des comparaisons par disciplines et par langues ${ }^{(9)}$. Dans une démarche comparative digne de ce nom, les disciplines et les langues-cultures ont besoin d'être étudiées pour elles-mêmes et non comme repoussoirs par rapport à des disciplines considérées comme étalons scientifiques, ou par rapport à une langue-cible idéale. Un troisième principe consiste, pour l'analyste du discours scientifique, dans la prise en compte des rapports de force et des formes d'inégalité (entre langues, entre disciplines) de manière à pouvoir comprendre les phénomènes de domination, d'interactions et d'influence, de censure, etc. Enfin, last but not least, il est important de fonder l'approche sur un paramétrage suffisamment fin : comparer des disciplines a pu être utile à un certain moment - et le reste dans une certaine mesure -, mais il y a trop de variation intra-disciplinaire pour que l'on en reste là ; d'où la nécessité d'avancer sur deux fronts :

- mieux considérer le lien entre disciplines et institutions, pour comprendre comment une discipline est structurée, au niveau international ou national, en la situant dans son histoire ; ajoutons que cette histoire institutionnelle ne trouve son sens qu'en fonction des évolutions scientifiques et épistémologiques des disciplines elles-mêmes ;

- se placer au niveau (sous-)disciplinaire le plus précis possible, comprenant déjà des déterminations de démarche ou d'objet : non pas « la linguistique » mais, par ex. «la phonétique expérimentale»; non pas « la sociologie » mais « la sociologie des institutions », etc.

En ce qui concerne cette fois la diversité générique, il reste à affiner la catégorisation, qui doit, dans la mesure du possible, intégrer d'autres critères que les seules mentions habituelles. Le linguiste Halliday, dans un article déjà ancien datant de 1988, intitulé "On the Language of Physical Science » fournit quelques clés toujours utiles pour compléter notre approche de la diversité des genres scientifiques. Il rappelle que le langage scientifique renvoie à un espace sémiotique qui se caractérise par une forte variabilité, aussi bien lorsqu'on le considère à un moment donné de son évolution que lorsqu'on l'envisage dans sa continuité diachronique. La diversité des genres peut, toujours selon Halliday, être expliquée en termes de champ, de tonalité et de mode. Le champ concerne l'aire disciplinaire, les sujets de connaissances considérés, la problématique scientifique, etc. Cette notion de champ est intéressante, parce qu'elle permet

(9) Le projet Scientext, réalisé au sein du Lidilem dans le cadre d'une ANR Corpus, a contribué au développement de corpus de textes scientifiques :http ://scientext.msh-alpes.fr/scientext-site/spip.php ?article 1 
de sortir d'une conception étroite de la discipline, là encore trop souvent entendue comme communauté fermée. La «tonalité » concerne la manière dont se configure la communication scientifique : au sein d'un même groupe (par ex. des spécialistes pour des spécialistes) ou d'un groupe à un autre (par ex. des enseignants pour des étudiants). Enfin, le mode concerne le canal (oral ou scriptural), le degré plus ou moins formel du langage utilisé, et l'organisation rhétorique liée aux enjeux de communication (expositive, hortative, polémique, etc.).

Concluons, pour finir, sur les précautions méthodologiques que doit prendre le chercheur pour éviter d'hypertrophier indûment un facteur de variation au détriment des autres ; il est essentiel, en effet, de ne pas considérer de manière univoque un facteur explicatif quelconque de la variation des discours scientifiques, sans le mettre en perspective en le considérant au sein de sous-systèmes complexes (linguistiques, historiques, épistémologiques). Une approche multidimensionnelle implique des collaborations pluridisciplinaires, prenant en compte trois grandes familles de paramètres :

- les paramètres liés aux systèmes linguistiques : ce n'est pas en partant d'une comparaison de marques de langue à langue qu'on peut progresser, mais en posant la question plus générale de la manière dont, par exemple, est mis en scène, dans les deux langues, l'effacement énonciatif, au sein de tel genre scientifique; il est important de procéder à des comparaisons de systèmes ou des micro-systèmes comparables ; cela paraît aller de soi, mais comme c'est compliqué, c'est finalement assez peu fait : la tendance est souvent de se limiter à la comparaison de marques prototypiques ; ajoutons les facteurs diachroniques : le fait de repérer des je dans un texte ne permet pas, on l'a vu, à lui seul de gloser sur la plus ou moins grande présence de l'auteur; la routinisation d'un dispositif énonciatif comme le nous académique en français ne nous autorise pas non plus à élucubrer sur la dimension plus ou moins personnelle des écrits analysés, mais simplement sur le degré d'intériorisation de normes propres à une époque donnée, ou sur leur peu d'évolutivité ; il nous faut donc intégrer plus précisément la dimension historique des usages linguistiques dans l'écrit scientifique.

- les paramètres liés aux systèmes culturels et aux normes éditoriales : il est nécessaire de mieux distinguer les normes culturelles et éditoriales des éléments proprement linguistiques, même s'il y a des interactions évidentes entre les deux aspects au plan textuel; cette distinction est d'autant plus nécessaire que la standardisation à l'œuvre dans le domaine de l'écriture scientifique tend à peser lourdement sur les évolutions culturelles, alors que les systèmes linguistiques évoluent beaucoup plus lentement; la textualité est à l'interface des dimensions linguistiques et éditoriales.

- les systèmes d'élaboration de la connaissance : cette troisième forme de médiation est encore plus souvent oubliée, dans les études portant sur l'écrit scientifique ${ }^{(10)}$. L'accent porté depuis Swales et ses épigones aux communau-

(10) Pour une approche du discours scientifique prenant en compte les facteurs épistémologiques, voir Grossmann (dir.) (2010). 
tés de discours a eu tendance à survaloriser les logiques identitaires - qui existent, bien entendu, mais sont aussi à relativiser, surtout à l'ère des échanges mondialisés. La question des disciplines s'inscrit dans le cadre plus large des dispositifs visant à élaborer de la connaissance, donc à travers les cadres épistémologiques que se donne le chercheur ou dont il hérite ; une telle conception permet seule de comprendre qu'au sein d'une même discipline, il puisse y avoir de la variation, celle-ci ouvrant aussi au changement et à l'innovation.

\section{Références}

BLOCH, J., \& CHI, L. (1995) : «A comparison of the use of citations in Chinese and English academic discourse », in D. Belcher \& G. Braine (Eds.), Academic writing in a Second Language, Norwood, NJ : Ablex, 231-274.

Boch, F. \& GROSSMANN, F. (2002) : «Se référer au discours d'autrui, quelques éléments de comparaison entre experts et néophytes », Enjeux, 54, 41-51

Bourdieu, P. (1984) : Homo Academicus, Paris, Éd. Les Éditions de Minuit, coll. « Le sens commun ».

Cavalla, C. \& Grossmann, F. (2005) : «Caractéristiques sémantiques de quelques "Noms scientifiques" dans l'article de recherche en français », AkademiskProsa, 2, Romanskinstitutt, Universitetet i Bergen, 47-59.

CLYNE, M. (1987) : «Cultural differences in the organization of academictexts. English and Germann », Journal of Pragmatics, vol. 11, 211-247.

- (1991) : «The Sociocultural Dimension : The Dilemma of the Germanspeaking Scholar », in Schröder, Hartmut et al. (eds) : Subject-oriented Texts. Language for Special Purposes and Text Theory, Berlin : de Gruyter, 49-67.

CONNOR, U. (2002) : «New directions in contrastive rhetoric », TESOL Quarterly, $36: 493-510$.

DAHL, T. (2004) : « Textual metadiscourse in research articles : a marker of national culture or of academic discipline? », Journal of Pragmatics, 36 (10), 1807-1825.

FEYERABEND, P. (1975) : Contre la méthode. Esquisse d'une théorie anarchiste de la connaissance. Paris : Seuil.

FLøтTUM, K. (2004) : «La présence de l'auteur dans les articles scientifiques : étude des pronoms je, nous et on », in A. Auchlin, E. Rouletet J.-M. Adam, Structures et discours. Mélanges offerts à Eddy Roulet, Québec, Nota bene, 404-414. 
Fløtтum, K., Dahl, T., Kinn, T., Gjesdal, A.-M. et Vold, E. T. (2007) : «Cultural identities and academic voices », in K. Fløttum (éd.), Language and Discipline Perspectives on Academic Discourse, Cambridge, Cambridge Scholars Publishing, 14-39.

Fløttum, K., JonAsson, K. et NorÉEn, C. (2007) : On, pronom à facettes, Bruxelles, De Boeck.

GALtunG, J. (1985) : Struktur, Kultur und intellektueller Stil. Einvergleichender Essay über sachsonische, teutonische, gallische und nipponischeWissenschaft ", in Wierlacher, Alois (eds.) : Das Fremde und das Eigene, München : Iudicum, 151-193.

GNUTZMANN, C. (1989) : «Sprachliche Indikatoren zur Explizierung von Zielsetzungenim Englischen und Deutschen », conférence tenue lors du $9^{\mathrm{e}}$ congrès du IDV à Vienne, manuscrit non publié.

GrossmanN, F. (2010) : L'auteur scientifique, des rhétoriques aux épistémologies. Présentation du vol 4, $\mathrm{n}^{\circ} 3$ de la Revue d'anthropologie des connaissances.

- (2010) (dir.) : «Les discours scientifiques : des marques linguistiques aux épistémologies », Revue d'Anthropologie des Connaissances, vol.4, $\mathrm{n}^{\circ} 3$.

Guilhaumou, J. \& MALdidier, D. (1986) : «Effets de l'archive. L'analysede discours du côté de l'histoire ", Langages, 81 : 43-56.

HALLYDAY, M.A.K. (1973) : Explorations in the functions of language, London, Edward Arnold.

- (1988) : «On the language of Physical Science », in Mohsen Ghadessy (ed.) Registers of Written English : Situational Factors and Linguistic Features, (OLS), London : Pinter, 162-178.

HyLAND, K. (2005) : Metadiscourse : Exploring Interaction in Writing. London : Continuum.

KAPLAN, R. (1966) : «Cultural thought patterns in intercultural education », Language Learning, 16.1-2, 1-20.

- (1972): The anatomy of rhetoric, Philadelphia/Pennsylvania : The Center for Curriculum Development.

KuHN, Th. (1962) : La structure des révolutions scientifiques. Paris : Flammarion.

LOFFlER-LAURiAN, A.-M. (1980) : «L'expression du locuteur dans les discoursscientifiques “je”, "nous" et "on" dans quelques textes de chimie et de physique », Revue de linguistique romane, 44, 135-157.

— (éd) (1983) : «Les discours scientifiques », Études de linguistique Appliquée, 51.

Maingueneau, D., CossutTA, F. (éds) (1995) : « L'analyse des discours constituants », Langages, 117, 112-125.

MORENO, A.I. (1997) : « Genre constraints across languages : causal metatext in Spanish and English Research Articles », ESP Journal, 16 (3), 161-179.

DE NUCHÈZE, V. (1998) : «Approches pragmatico-énonciative du discours de recherche (à l'usage des apprenants-chercheurs) », Lidil, 17, 27-40. 
Olivesi, S. (2007) : Référence, déférence : une sociologie de la citation. Paris : L'Harmattan.

PÊCHEUX, M. (1990) : L'inquiétude du discours, textes choisis et présentés par Denise Maldidier. Paris : Editions des Cendres.

PONTILLE, D. (2004) : La signature scientifique. Une sociologie pragmatique de l'attribution. Paris :CNRS Éditions.

- (2007) : «Matérialité des écrits scientifiques et travail de frontières : le cas du format IMRAD », in P. Hert et M. Paul-Cavallier (eds.), Sciences et frontières, Fernelmont, E.M.E., 229-253.

POUDAT, C. (2006) : Etude contrastive de l'article scientifique de revue linguistiquedans une perspective d'analyse des genres, Thèse en sciences du Langage, Université d'Orléans.

REUTER, Y. (1998) : «De quelques obstacles à l'écriture de recherche », Lidil, $17,11-23$.

REUTNER, U. (2010) : «De nobis ipsis silemus ?Les marques de personne dans l'article scientifique », Lidil, 41, 79-102.

RINCK, F., BOCH, F., GROSSMANN, F. (2007) : «Quelques lieux de variation du positionnement énonciatif dans l'article de recherche », in A. Millet, M. Rispail, P. Lambert et C. Trimaille (eds.), Variations au cour et aux marges de la sociolinguistique. Hommages à Jacqueline Billiez, Paris, L'Harmattan, 285-296.

Rosental, C. (2009) : "Anthropologie de la démonstration », Revue d'Anthropologie des Connaissances, 3 (2), 233-252.

SAVILlE-Troike, M. (1982) : The Ethnography of Communication, Oxford : Blackwell.

SWALES, J. M. (1990) : Genre Analysis : English in academic and research settings, Cambridge, Cambridge University Press.

SCHWARZE, S. (2008) : "Introduction : La notion de "style” par rapport au discours scientifique ", in Reutner et Schwarze (eds), Le style c'est l'homme? Unité et diversité du discours scientifique dans les langues romanes, Frankfurt a.M., Peter Lang, 1-22.

SPILLNER, B. (1989) : «Stilelemente im fachsprachlichen Diskurs », in Dahmen, Wolfgang/Holtus, Günter/Kramer, Johannes (eds.) : TechnischeSprache und Technolekte in der Romania, Tübingen : Narr, 2-19.

SCHRÖDER, H. (1995) : «Der Stilwissenschaftlichen Schreibens zwischen Disziplin, Kultur und Paradigma - Methodologische Anmerkungen zur interkulturellen Stilforschung ", in Stickel, Gerhard (ed.) Stilfragen, Berlin/New York : de Gruyter, 150-180.

TOUATI, P. (2010) : « De la médiation épistolaire dans la construction du savoir scientifique. Le cas d'une correspondance entre phonéticiens », Revue d'anthropologie des connaissances, vol.4, $\mathrm{n}^{\circ} 3$, 451-475.

Tutin, A., Grossmann, F., FAlaise, A., Kraif, O. (2009) : Autour du projet Scientext : étude des marques linguistiques du positionnement de l'auteur dans les écrits scientifiques, Journées « Linguistique de corpus », Lorient.

TUTIN, A. (2010) : «Dans cet article, nous souhaitons montrer que... Lexique 
verbal et positionnement de l'auteur dans les articles en sciences humaines. Enonciation et rhétorique dans l'écrit scientifique », Lidil, 41, 15-40.

VALERO-GARCES, C. (1996) : «Contrastive ESP Rhetoric : metatext in spanishenglish economics texts », English for SpecificPurposes, 15(4), 279-294.

VANDE KOPPLE, W. (1985) : «Some exploratory discourse on métadiscours », College Composition and Communication, (36), 82-93.

lidil

Pratiques de formation à la lecture-écriture des adultes en parcours d'insertion. Enjeux didactiques et institutionnels

M.-H. Luis, S. Majaji, C. Ciappara-Diaz, J.-M. Besse : Illettrisme, évaluation et formation : réflexions sur une approche européenne

C. Portefin : Adaptation des formateurs en milieu professionnel dans un environnement en mutation

H. Adami, V. André : Formation aux écrits professionnels : vers une sociolinguistique impliquée

M.-C. Guernier : Les contenus linguistiques dans les référentiels et les discours des formateurs. Définitions, conceptions et références

V. Leclercq, A. Vicher : Etude sur les usages des référentiels dans la formation linguistique de base

F. Chnane-Davin, C. Lafarge : Interactions orales et réflexion sur la langue dans la réécriture chez l'adulte

M. Niwese : Balises pour un atelier d'écriture adapté à un public adulte en difficulté

B. Balas : La copie de texte comme outil d'évaluation des représentations de la langue écrite par l'adulte dans son parcours d'apprentissage

M.-H. Lachaud, D. Rehaili : Discours des apprenants sur les effets de la formation à l'écrit

V. Rivière : Regards socio-didactiques sur les pratiques de formation linguistiques pour les adultes en parcours d'insertion

Renseignements - commandes :

Revues/Ellug

Université Stendhal, BP 25 - 38040 Grenoble Cedex 9

brigitte.pautasso@u-grenoble3.fr — http://w3.u-grenoble3.fr/ellug 